\title{
A Distributed Mobility Management Framework for 5G Converged Networks
}

\author{
Kyounghee Lee ${ }^{1, *}$ and No-Ik Park ${ }^{2}$ \\ ${ }^{1}$ Department of Computer Engineering, Pai Chai University, Daejeon, Korea \\ ${ }^{2}$ Hyper-connected Communication Research Lab., ETRI, Daejeon, Korea \\ ${ }^{1}$ leekhe@pcu.ac.kr, ${ }^{2}$ nipark@etri.re.kr
}

\begin{abstract}
A mobility management issue is surfacing again as it is expected that mobility requirements will be very essential and vary with application scenarios in the future $5 G$ networks. An important research direction on this area is to design a flat and distributed control architecture to achieve scalability enough to be applied into the wide-scale real networks. In this paper, we propose a distributed mobility management framework for evolving networks toward $5 G$. The main idea is to separate location management from data traffic control during handling a node's handover. By being divided from location update to be propagated throughout whole network, handover control process can be localized and accelerated in a distributed manner. To apply different control operations according to handover types is another important feature of our approach. Experimental results from our simulation study show that the proposed framework considerably outperforms a well-known MM protocol, Proxy Mobile IPv6, in the aspects of handover latency, packet loss and dispersion of control overhead.
\end{abstract}

Keywords: IP Mobility, 5G Network, Distributed Mobility Management

\section{Introduction}

Currently, communication network is evolving toward 5G which is not just a new generation of mobile cellular network but a new kind of network embracing all features required for future Internet. Researches on radio communications have suggested some core technologies for $5 \mathrm{G}$ networks such as massive multiple-input multiple-output (MIMO) and millimeter wave (mmWave) transmission. Those technologies could realize high-speed data rate of tens and hundreds Gbps at the mobile network edge. Another important challenge in the slower lane is to draw a new architecture for 5G core network. Many experts expect it would be a common single architecture integrating various mobile and fixed access technologies.

Through activities of the Focus Group IMT-2020 in ITU-T, several interesting issues are being discussed to illustrate a common architecture of $5 \mathrm{G}$ core network, including separation of control and user planes, network slicing, and support of different levels of mobility (called "on-demand mobility") [1-2]. A mobility issue is surfacing again as it is expected that mobility requirements for user devices will be essential and vary with application scenarios.

On observing IP mobility management (MM) technology, recent researches can be classified with two categories: a scalable MM architecture and differentiation of mobility services. The first category is related to how to put $\mathrm{MM}$ architecture to practical use in the real networks, especially in 5G networks. One of notable approaches in this field is distributed MM (DMM) to design a scalable control architecture by moving mobility functions from the core to the edge of network. It is not newly issued but enters a new

${ }^{*}$ Corresponding Author 
phase as network softwarization technologies such as software defined networking (SDN) and network functions virtualization (NFV) are being considered for core technologies of $5 \mathrm{G}$ networks.

The second category covers the on-demand mobility issue to support multiple levels of mobility requirements depending on various future application scenarios. A lot of new services based on Internet of things (IoT) and Big Data have recently appeared and they require new functions of network so that each service can be supported with individual and differentiated control. Researches in this field seem to be their infancy and some existing approaches for flow-based mobility control [3-4] may be starting points to achieve their goals.

In this paper, we design an IP-based DMM framework considering new features and requirements of evolving $5 \mathrm{G}$ networks. Our framework focuses on increase of scalability achieved by mobility information distribution and localized handover control. A key idea is to entirely separate location management from data traffic control on handovers of mobile nodes (MNs). In our framework, location information is only used to know a MN's current location when a new data session is established by another node in different network. Therefore, such location information does not have to be updated in a realtime manner during handover control is dealt with. This enables handover control in our framework to be locally handled and accelerated. Different control operations according to handover types (referred to FB and FF types) are also introduced to improve control efficiency of the proposed DMM framework.

The rest of this paper is organized as follows. In Section 2, we look around some related researches on DMM. In Section 3, we describe the proposed DMM framework with some design considerations. Experimental results obtained from simulation study are given in Section 4. Finally, we conclude the paper in Section 5.

\section{Related Work}

In [5], three main families of the current DMM approaches have been described and analyzed: PMIPv6-based, SDN-based and routing-based DMM solutions.

Proxy Mobile IPv6 (PMIP6), a standard protocol of IETF, is a network-based MM approach without requiring any mobility functions to end-hosts $[6,17]$. In the PMIPv6 network, there exists a proxy, called Mobile Access Gateway (MAG), handling handover operations on behalf of MNs. An anchoring unit, called Local Mobility Anchor (LMA) manages binding information of MAGs associating with MNs and handles traffic control by tunneling with those MAGs. In PMIPv6-based DMM approaches [7-8], MAGs are generally extended to configure tunneling between themselves instead of LMA-to-MAG tunneling. To distribute location information of MNs, some approaches enable the Proxy Binding Update (PBU) process to be handled by two adjacent MAGs [7]. Those approaches, called fully distributed $\mathrm{MM}$, do not require any functions of the existing LMA in the PMIPv6 framework. Other approaches, called partially distributed MM, reduce LMA's functions to participate only in location management of MNs [8].

SDN-based DMM solutions are similar to PMIPv6-based ones. The difference is that all handover operations such as location update and traffic control are handled by the SDN control layer. In the SDN-based approach described in [5], a MAG is replaced with an OpenFlow switch [14] called DMM gateway (DMM-GW). The network controller (NC) recognizes a MN's handover when the MN's Router Solicitation (RS) message has delivered from a new DMM-GW area. Then the NC updates flow tables of two DMMGWs related to the MN's handover so that packets are forwarded to the MN's new location. Packet forwarding between DMM-GWs does not need to be implemented with IP tunneling method.

The basic concept of routing-based DMM approaches is to remove any anchor node from the network. Those approaches make all routers re-establish a new routing map 
whenever a MN moves between different DMM-GWs. In [9], the idea is achieved by enabling Border Gateway Protocol (BGP) [10] on DMM-GWs so as to propagate upward the changes of their links to their BGP peer routers. Though it is a fully distributed MM solution, we cannot ignore that signaling overhead from frequent BGP updates and the consequential additional latency on handover control may become considerably high as the number of routers increases.

\section{Proposed DMM Framework}

One of challenges in $5 \mathrm{G}$ core network design is to integrate various access technologies into a single architecture. It may not cover only new radio for $5 \mathrm{G}$ but also the existing $3 \mathrm{G} / 4 \mathrm{G}$ and wired technologies as well. A feasible idea is to put a convergence gateway $(\mathrm{C}-\mathrm{GW})$ at the core network edge so that it accommodates heterogeneous access types and provides a common interface to the core network. Figure 1 shows the concept of C-GW for $5 \mathrm{G}$ networks, being developed by Electronics and Telecommunications Research Institute (ETRI). The proposed DMM framework has been designed with functions and operations to be deployed to those C-GWs.

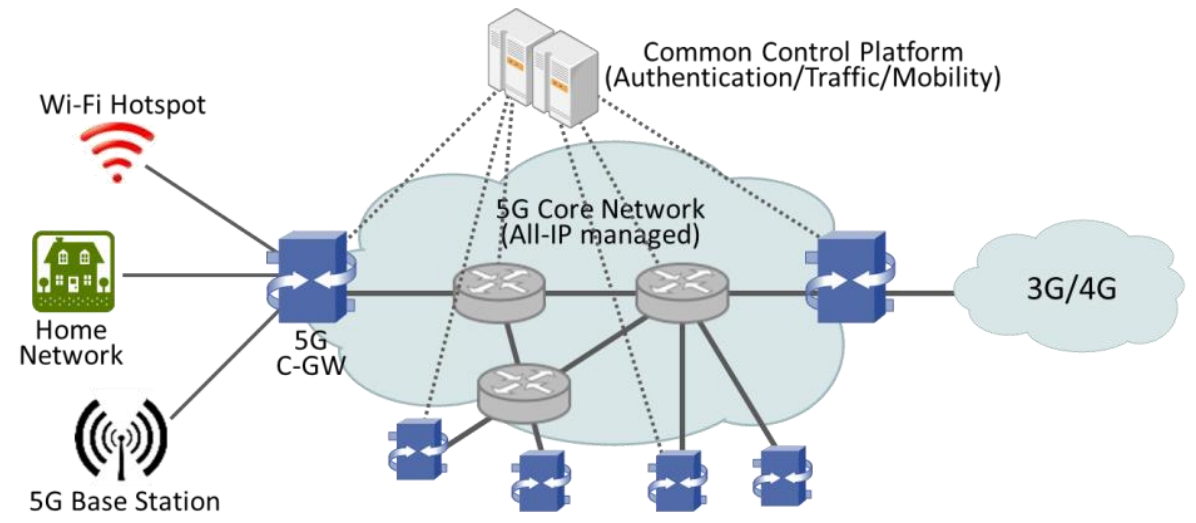

Figure 1. Conceptual diagram of 5G Convergence Gateway

In [11], we have proposed a host identification scheme based on the Uniform Resource Identifier (URI) addressing system [15]. Binding information of a MN's host identifier (i.e., URI) and IP address can be fully distributed over the networks with the hierarchical structure of Domain Name System (DNS) [16]. Since this location information is only used when a new data session is established for a $\mathrm{MN}$, the requirement of realtime update due to the MN's handover can be escaped. It is why traffic control for a handover can be locally handled regardless of location update process. We take this URI-based location management mechanism for our DMM framework in this paper.

We have also introduced a classification of two handover types from analysis of users' movement pattern and network environment: Forward-and-Backward (FB) type and Forward-and-Forward (FF) type. FB type handover is defined as that a MN changes its connection to a new access network during the first handover and returns back to the previous access network through the second handover. On the other hand, FF type handover is defined as that a MN moves to the third access network through the second handover after performing the first forward handover. More details on this can be found in [11]. Our DMM framework is designed with consideration of different control schemes according to these handover types.

\subsection{Intra-C-GW Handover Control}

As mentioned above, a C-GW can connect multiple types of access links which generally belong to different routing domains (or subnet). Figure 2 illustrates the intra-C- 
GW handover control process when a MN moves between two subnets connected to a single $\mathrm{C}-\mathrm{GW}$ via different interfaces.

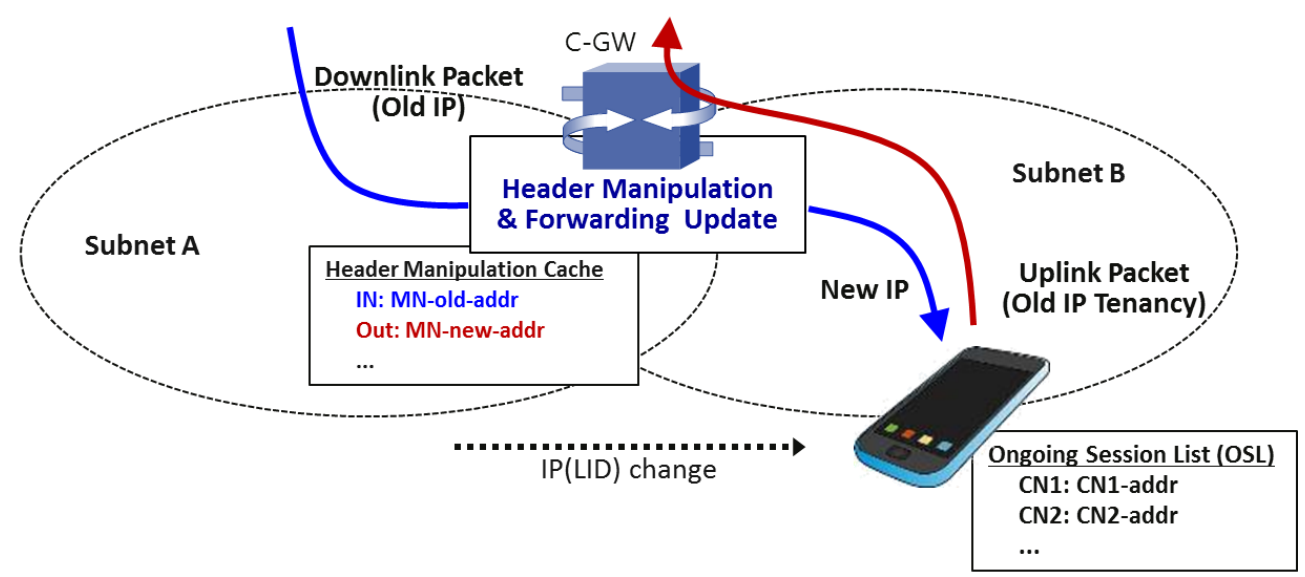

Figure 2. Intra-C-GW Handover Control

For downlink traffic delivered to a MN, the network-based control is applied. As shown in Figure 2, when a MN moves from Subnet A to Subnet B and changes its location identifier (LID) with newly allocated IP address in Subnet B, C-GW starts overwriting a destination address in the header of each packet destined to the MN's old IP address with a new IP address (i.e., header manipulation). Then the outgoing port of those packets is changed toward Subnet B so that they are delivered to the MN's new location (i.e., forwarding update).

If the $\mathrm{MN}$ is participating in an on-going session which has been established before a handover, uplink packets belonging to the session should be specially handled to maintain session continuity. In this case, the MN configures the source address of those packets with its old IP address used in Subnet A. To do this, the MN should manage its own Ongoing Session List (OSL) which consists of the addresses of CNs involved to on-going sessions. This is called old IP address tenancy in our DMM framework.

\subsection{Inter-C-GW Handover Control (FB Type)}

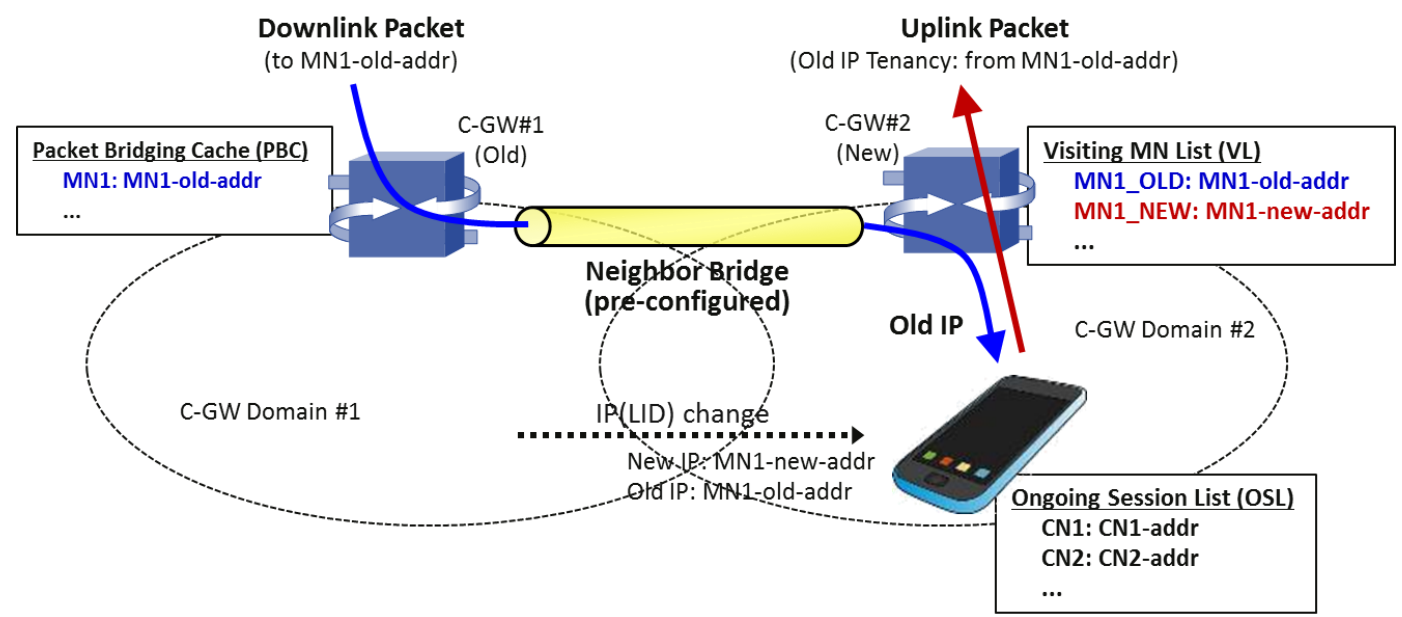

Figure 3. Inter-C-GW Handover Control (FB type: forward)

The first half to control a FB type handover is to deal with the first handover (forward). Figure 3 shows the handover control process when a MN moves from C-GW\#1's domain 
to C-GW\#2's domain. As shown in the figure, after a handover, the MN changes its LID with a newly allocated IP address from C-GW\#2's domain. In this situation, downlink packets from outside destined to the $\mathrm{MN}$ are delivered to $\mathrm{C}-\mathrm{GW} \# 1$ since their destination addresses are the MN's old IP address. To deliver those packets to the MN's current location, $\mathrm{C}-\mathrm{GW} \# 1$ forwards them to $\mathrm{C}-\mathrm{GW} \# 2$ through a pre-configured neighbor bridge. After passing the neighbor bridge, those packets are finally delivered to the MN's old IP address. Since there is no change in those packets' addresses, the on-going sessions' continuity can be maintained.

Each C-GW has to manage its own Proxy Binding Cache (PBC) and Visiting MN List (VL) to perform such neighbor bridging operations. A PBC is managed for each neighbor bridge and temporarily stores old IP addresses of MNs which have just moved to the neighbor C-GW domain connected with the neighbor bridge. A VL is generated for each C-GW and used to check if the destinations of packets from neighbor bridges are really located in its domain. Control for uplink packets belonging to an on-going session is equivalent to that of the intra-C-GW handover case describe earlier. To provide session continuity, each MN manages an OSL and the old IP address tenancy scheme is also applied for on-going sessions.

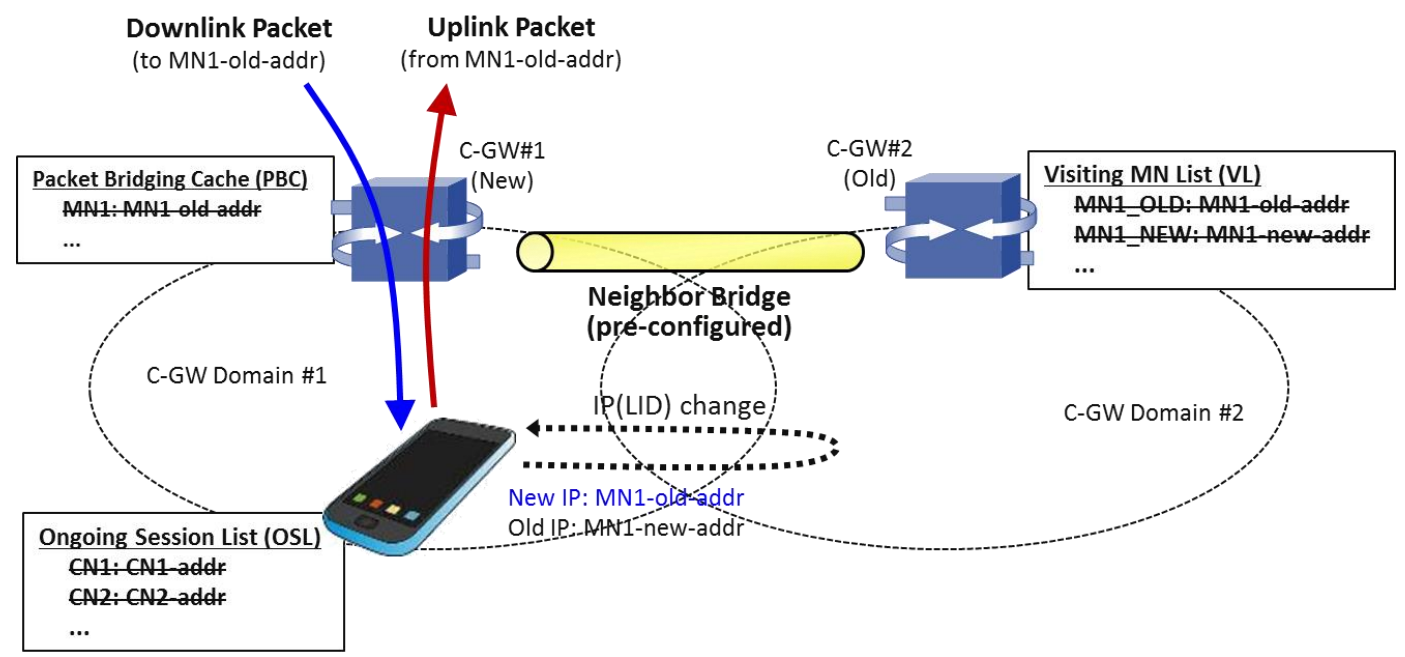

Figure 4. Inter-C-GW Handover Control (FB type: backward)

Figure 4 illustrates the second half of FB type handover control. If a MN moves back to the previous location (i.e., C-GW\#1's domain in the figure), the second handover (backward) occurs. Since the MN's old IP address (MN1-old-addr) used in C-GW\#1's domain has been borrowed by the IP address tenancy scheme, it can be immediately reallocated to the MN after a backward handover. Subsequently, C-GW\#1 deletes the reallocated IP address from its PBC to inactivate neighbor bridging for the packets destined to the IP address. The MN also resets its OSL to be null because it has took back the old IP address used for on-going sessions. On the other side, C-GW\#2 deletes unnecessary entries for the MN from its VL since the MN has already left it domain.

As a result, the MN returns to the initial state before the first handover of FB type had occurred. We need to consider if a $\mathrm{MN}$ has made new sessions while residing in $\mathrm{C}$ GW\#2's domain. If so, the first half of FB type control process shown in Figure 3 should be performed reversely (for a movement from C-GW\#2 to C-GW\#1) to support those ongoing sessions' continuity. 


\subsection{Inter-C-GW Handover Control (FF Type)}

The control process of FF type handover is depicted in Figure 5. This process is a set of proactive actions to prepare a situation that a $\mathrm{MN}$ moves again to another $\mathrm{C}-\mathrm{GW}$ domain (i.e., the third C-GW's domain which is not expressed in Figure 5) after the first half of FB type control process has been completed. Thus this process needs to be initiated when it is strongly expected that the MN's next movement is not towards its previous location (i.e., C-GW\#1's domain). The key idea is to restore the MN's state to before a forward handover of FB type so that the next handover is regarded as the first handover from the initial state. This can be done in host-based control manner, referred to route optimization, which has been introduced in [11].

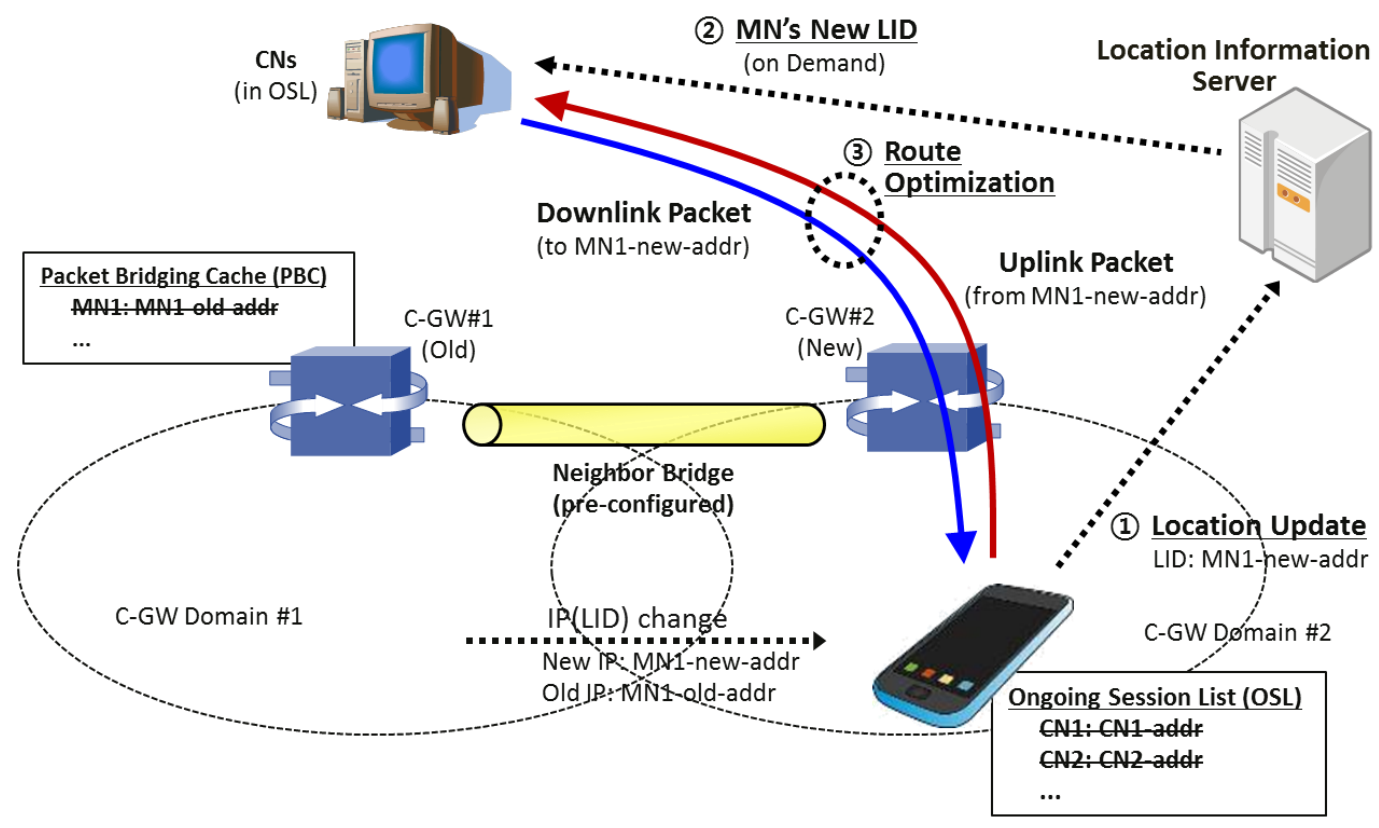

Figure 5. Inter-C-GW Handover Control (FF type)

A MN should update its LID registered in Location Information Server (LIS) whenever it moves among C-GW domains and changes its IP address. As said before, this is not a part of handover control and realtime update is not mandatory. When a $\mathrm{CN}$ knows a new LID of the MN, it can change the destination IP address of packets to be directly delivered to the $\mathrm{MN}$ via $\mathrm{C}-\mathrm{GW} \# 2$. Subsequently, the $\mathrm{MN}$ can also send packets to the $\mathrm{CN}$ with a new source IP address. The MN deletes the CN's entry from its OSL since the old IP address tenancy is not more necessary. The MN's entry in C-GW\#1's PBC will be expired or deleted to inactivate neighbor bridging. Finally, a route between the MN and the $\mathrm{CN}$ is optimized.

\section{Experimental Results}

In this section, we present some experiments results from our simulation study. Two experiment scenarios have been applied to evaluate control efficiency (i.e., handover latency and packet loss) and control overhead dispersion, respectively. The NS3 network simulator [12] was used for the first experiment and a Python script program was written to monitor control messages for tens of thousands of MNs. Figure 6 shows the simulation network topology used in our experiments.

There are four domains where a $\mathrm{MN}$ moves around the simulation network. For comparative study, we set each domain to be managed by MAG in PMIPv6 or a C-GW in our framework, which is located at the domain's gateway. LMA in PMIPv6 and LMS in 
our framework are placed in another domain which a $\mathrm{CN}$ resides. We have configured four neighbor bridges between C-GWs as shown as a ring structure in the figure. Those are used only for our DMM framework.

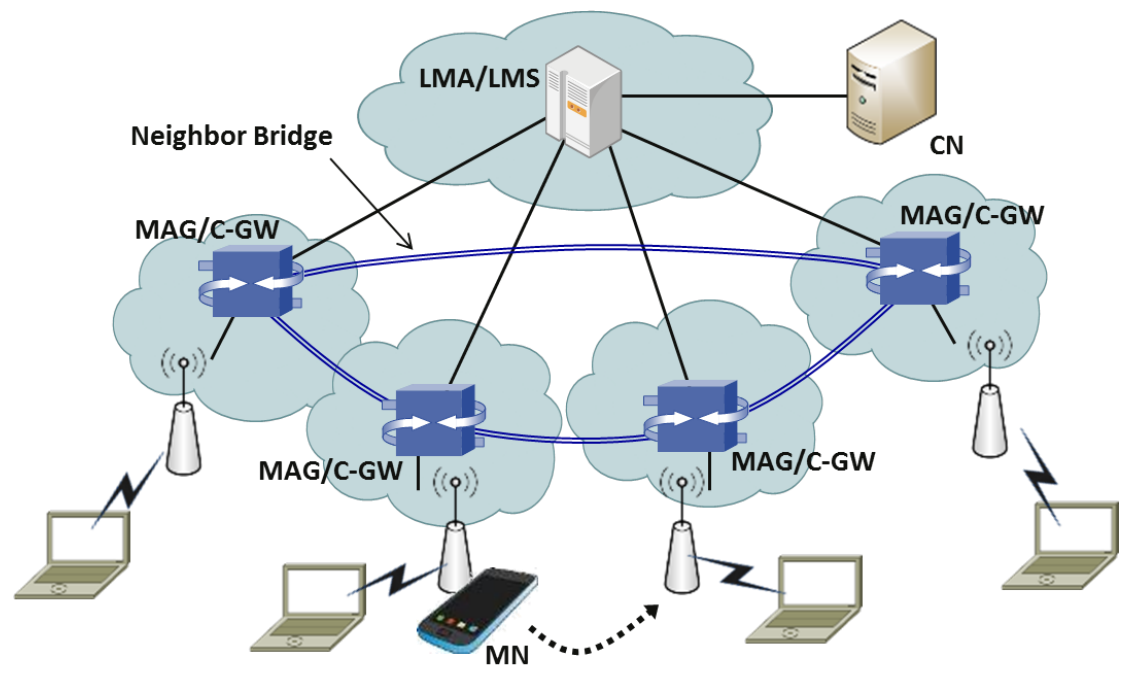

Figure 6. Simulation Network Topology

Since the NS3 simulator does not impose a message processing time on each node, we set such a delay at LMA/C-GW using a random variable following a normal distribution. The normal distribution average was calculated by dividing the number of incoming control messages per second by the maximum message capacity per second at a node. We used one eighth of the average as an estimate of variance (according a heuristic approach). The maximum capacity of message handling at a node was configured to be $11.31 \mathrm{Mpps}$ which is known as the fastest message handling speed at a software switch using an Intel $10 \mathrm{GbE}$ network interface card (NIC) [13].

In the first experiment, the MN moves continuously across the network by randomly choosing one of neighbor MAG/C-GW domains. Other wireless nodes (i.e., laptops in the figure) do not move. The $\mathrm{CN}$ consistently sends data packets to the $\mathrm{MN}$ while it is moving. The transmission rate is $4 \mathrm{Mbps}$ since the packet size is 1024 bytes and the packet interval is 2 milliseconds (ms).
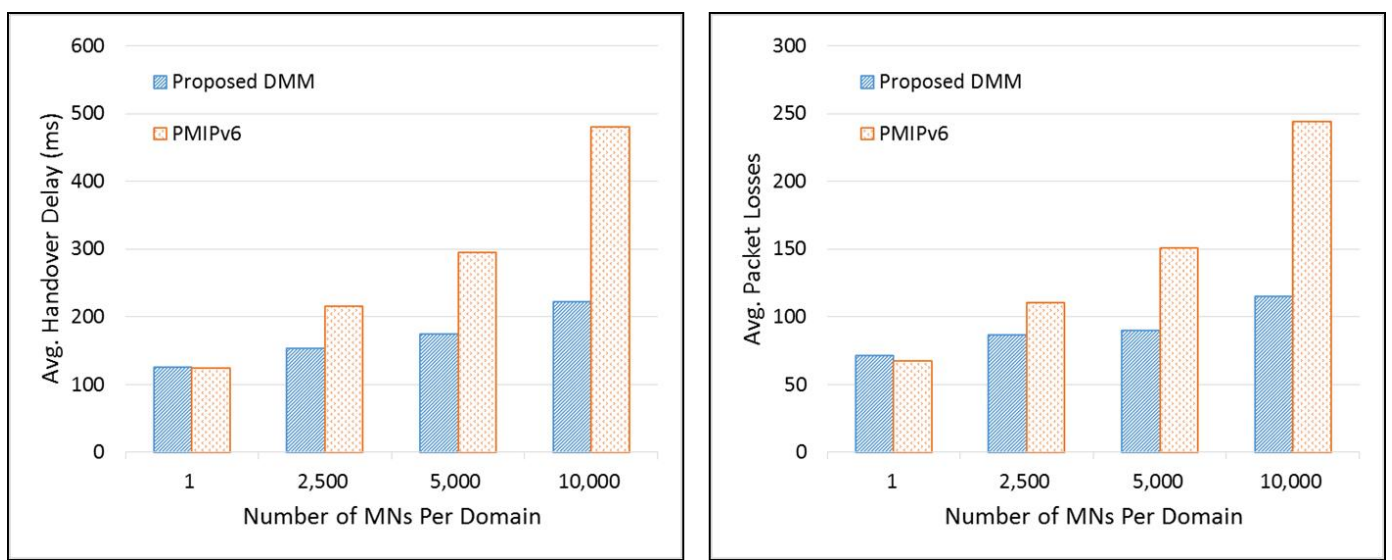

Figure 7. Average Handover Delay and Packet Loss

Figure 7 shows handover delay and number of packet losses due to a handover, which were measured from the simulation. As shown in the figure, those two evaluation factors 
of PMIPv6 appear slightly better than our DMM framework when the number of MNs is not high. This is because the proposed approach may require additional delay for neighbor bridging (i.e., more links in a traffic path). However, as the number of MNs increases, our approach distinctly outperforms PMIPv6. For example, when 10,000 MNs exist per a domain, those two factors appeared $481 \mathrm{~ms}$ and 244 losses in PMIPv6 and $223 \mathrm{~ms}$ and 115 losses in our approach, respectively. This performance advantage of the proposed mechanism can be explained by that the control overhead is distributed to four C-GWs at the network edge while it is concentrated to LMA in PMIPv6.
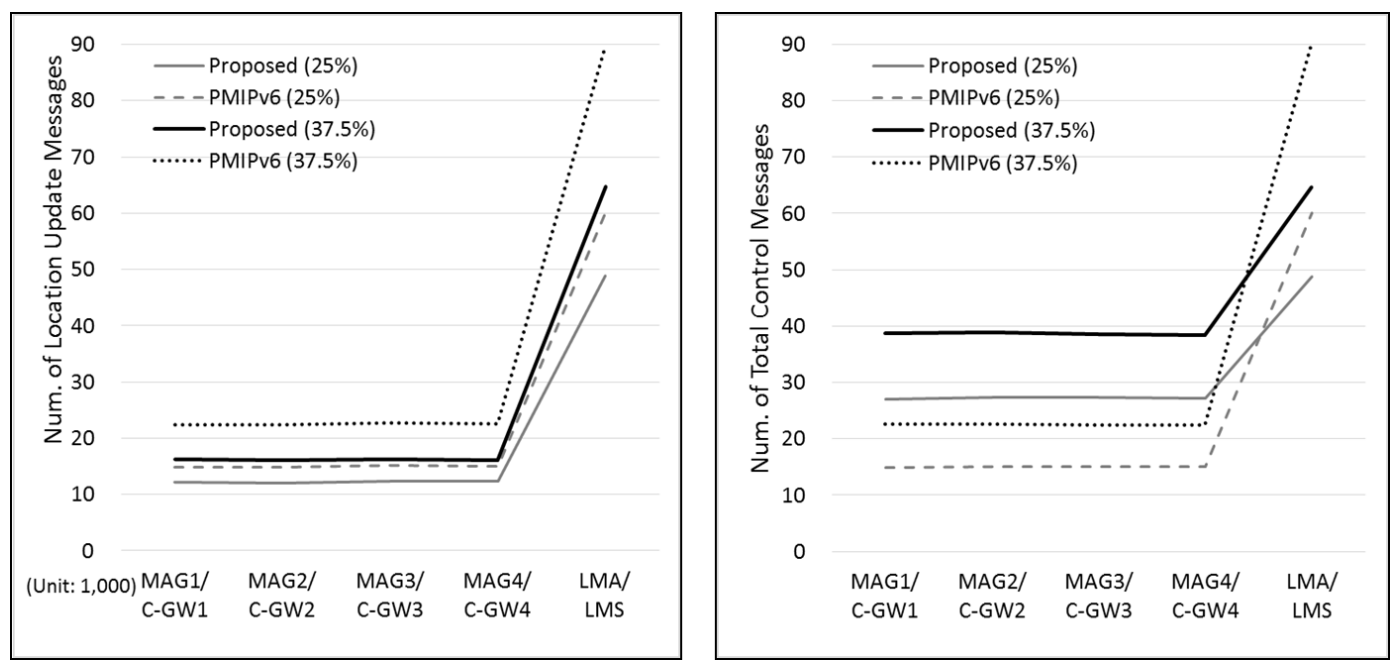

Figure 8. Dispersion of Control Messages

Figure 8 shows the results of the second experiment to evaluate how control overhead is dispersed over the network. At the start of this experiment, 10,000 MNs are located in each MAG/C-GW domain (totally 40,000 MNs in the network). The above results present the number of control messages delivered to each control node of PMIPv6 and the proposed DMM framework. The experiment was carried out for 30 seconds in two cases that $25 \%$ and $37.5 \%$ of MNs are moving every 5 seconds to a randomly selected one of their neighbor domains.

As shown in Figure 8, while the number of messages per MAG/C-GW is evenly distributed, it appears quite different at LMA/LMS. This is because all PBU/PBA (in PMIPv6) and Location Update/Ack (in our framework) messages should be handled by LMA/LMS. In the aspect of location management process, the number of messages distributed to C-GW/LMS in our approach is generally lower than that of MAG/LMA of PMIPv6. This can be understood by that a MN sometimes does not generate a Location Update message while it is handled by the FB type handover control process in the proposed framework.

When all control messages are considered, the results become different. The number of messages handled by each C-GW appears higher than that of MAG. This is caused by additional messages of handover notification, which are exchanged between adjacent $\mathrm{C}$ GWs to update their PBCs and to activate neighbor bridging. However, it is obvious those handover notification messages move around the network edge but not through the core network. It is notable that the number of messages at LMS is considerably lower than that of LMA. This represents that the control overhead to support mobility is not much concentrated to a specific node when the proposed DMM framework is applied. 


\section{Conclusion}

In this paper, we have introduced an IP-based DMM framework which was designed with consideration of new features and requirements of evolving $5 \mathrm{G}$ networks. The proposed framework separates location management from data traffic control on handling a MN's handover so that the necessary process is localized and accelerated. To apply different control operations according to handover types is another important feature of our approach to improve control simplicity and efficiency. We have presented some experimental results obtained from simulation study to evaluate performance of the proposed framework. Those results show that our approach distinctly outperforms PMIPv6 in handover latency and packet loss. Notably, we have showed that the control overhead to support mobility can be well-dispersed over the network when the proposed framework is applied.

\section{Acknowledgments}

This work was supported by Institute for Information \& communications Technology Promotion (IITP) grant funded by the Korea government, Ministry of Science and ICT and Future Planning (MSIP) (No. B0132-16-1005, Development of Wired-Wireless Converged 5G Core Technologies).

\section{References}

[1] Technical Document, "FG IMT-2020: Report on Standards Gap Analysis", TD 208, Focus Group IMT2020 in ITU-T, (2015).

[2] A. Yegin, D. Moses, K. Kweon, J. Lee and J. Park, “On Demand Mobility Management”, IETF InternetDraft, draft-ietf-dmm-ondemand-mobility-07, (2016).

[3] A. de la Oliva, C. J. Bernardos, M. Calderon, T. Melia and J. C. Zuniga, "IP Flow Mobility: Smart Traffic Offload for Future Wireless Networks", IEEE Communications Magazine, vol. 49, iss. 10, (2011), pp. 124-132.

[4] C. J. Bernardos, "Proxy Mobile IPv6 Extensions to Support Flow Mobility", IETF RFC 7864, (2016).

[5] F. Giust, L. Cominardi and C. J. Bernardos, "Distributed Mobility Management for Future 5G Networks: Overview and Analysis of Existing Approaches”, IEEE Communications Magazine, vol. 53, iss. 1, (2015), pp. 142-149.

[6] S. Gundavelli, K. Leung, V. Devarapalli, K. Chowdhury and B. Patil, "Proxy Mobile IPv6", IETF RFC 5213, (2008).

[7] C. J. Bernardos and J. C. Zuniga, "PMIPv6-based distributed anchoring", IETF Internet-Draft, draftbernardos-dmm-distributed-anchoring-08, (2016).

[8] C. J. Bernardos, A. de la Oliva and F. Giust, "A PMIPv6-based solution for Distributed Mobility Management", IETF Internet-Draft, draft-bernardos-dmm-pmip-07, (2016).

[9] P. McCann, "Authentication and Mobility Management in a Flat Architecture", IETF Internet-Draft, draft-mccann-dmmflatarch-00, (2012).

[10] Y. Rekhter, T. Li and S. Hares, "A Border Gateway Protocol 4 (BGP-4)", IETF RFC 4271, (2006).

[11] S. Han, M. Kim, K. Lee, K. Kim, H. Kim and W. You, "An IP Mobility Management Framework based on Handover Type Differentiation and Distributed Location Management", International Journal of Applied Engineering Research, vol. 10, no. 13, (2015), pp. 33583-33586.

[12] "NS-3 Network Simulator", available at: https://www.nsnam.org.

[13] P. Emmerich, D. Raumer, F. Wohlfart and G. Carle, "Performance characteristics of virtual switching", Proceedings of the IEEE 3th International Conference on Cloud Networking, Luxembourg, Luxemburg, (2014).

[14] ONF Standard, "OpenFlow Switch Specification", Version 1.1.0 Implemented, Open Networking Forum, (2011).

[15] T. Berners-Lee, R. Fielding and L. Masinter, "Uniform Resource Identifier (URI): Generic Syntax", IETF RFC 3986, (2005).

[16] P. Mockapetris, "Domain Names - Implementation and Specification", IETF RFC 1035, (1987).

[17] R. Wakikawa and S. Gundavelli, "IPv4 Support for Proxy Mobile IPv6", IETF RFC 5844, (2010). 


\section{Authors}

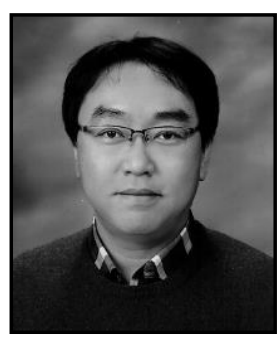

Kyounghee Lee, he received his B.S. degree in Computer Science from Kwangwoon University, Seoul, Korea, in 1999. He received his M.S. and Ph.D. degrees in Korea Advanced Institute of Science and Technology (KAIST), Daejeon, Korea, in 2000 and 2006, respectively. From 2006 to 2013, he was a senior researcher in Electronics and Telecommunications Research Institute (ETRI), Daejeon, Korea. Since 2013, he has been a faculty member of Department of Computer Engineering in Pai Chai University, Daejeon, Korea. His research interests include future network, mobile commuincations, Internet of Things, mobile software and real-time multimediae.

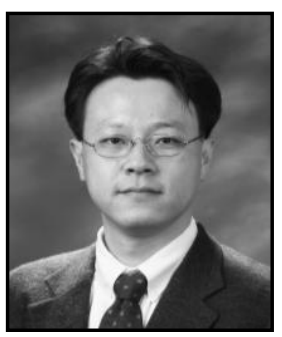

No-Ik Park, he received the B.E., M.E., and Ph.D. degrees in Industrial Engineering from Sung Kyun Kwan University, Korea, in 1993, 1995, and 2000, respectively. He joined Electronics and Telecommunication Research Institute (ETRI), Korea, in 2000. His main areas of research interest are network performance analysis, network engineering, QoS, IP mobility, 5G network, etc. He is currently Director of 5G Network Research Section in ETRI. 